\title{
Multicue Optimized Object Detection for Automatic Video Event Extraction
}

\author{
B. S. Daga ${ }^{1 *}$ and A. A. Ghatol ${ }^{2}$ \\ 'Computer Engineering Department, Fr. Conceicao Rodrigues College of Engineering, Mumbai - 400050, India; \\ bsdaga77@gmail.com \\ 2Dr Babasaheb Ambedkar Technological University, Lonere - 402103, India; \\ ashok.ghatol@gmail.com
}

\begin{abstract}
Objective: To demonstrate the video event extraction system, combining the multicue optimized object detection with video semantic modelling. Methods/ Statistical Analysis: The literature established video semantic model has been recreated with a limited model. The multicue optimized object detector has been employed substituting the primary object detector in the system. The multicue object detector provides the inter object spatio-temporal cue information for event detection and aids to the semantic model. This similitude system establishes the proof of concept for the idea of incubating the multicue object identifier within a video extraction system for performance augmentation. The system had been tested for two different event extractions from a combination of six videos. Findings: The testing results had been presented for the two target events demonstrating the multicue object detector performing in frame by frame manner, for the test videos. The system performance measured and presented in terms of precision and recall indicates encouraging enhancements in the baseline system accuracy. Application/Improvement: The novelty of the work presented lies in clubbing the most advanced multicue optimized object detection technology with a semantic model based video extraction system, and thus bringing together the benefits of spatio-temporal cue method with a semantic model system.
\end{abstract}

Keywords: Event Detection, Event Extraction, Multicue Object Identifier, Spatio-Temporal Cue, Video Content Modelling

\section{Introduction}

Undoubtedly the twentieth century had been the era of inventions and high end technological advancements for mankind. Many of the literature articles, online essays and reports suggest that the internet had been the top most invention of the twentieth century and would be the most valuable gift from this generation to all future generations. With the internet came the search engines, which offered meaningful access to vast information available online. We have also seen search engines maturing to the different levels of information type extractions, are it documents based, image based, video based, etc. And then, as one talk about internet and videos, there had been the rise of gigantic open access video collection sites, e.g. youtube.com. Nowadays one has many more online video collections to choose from such as, sports based, televisions, movies, etc. In short, a video is one of the best types of information compilations and we have innumerable of it available to choose and access through the internet.

The internet user's attention span is reducing with growing content availability and data speed. Thereby, as one narrows down to the problem of video searches, one understands that simply providing the related search topic videos won't be good enough going

${ }^{*}$ Author for correspondence 
further in future. The developers and video content providers need to understand and respect user's instant based interest to stay alive in the future age of information science. For example let's consider a case of a user seeking for a football match instance where the player strikes the goal. In most likely situations user is obviously not interested in spending time over watching the entire match feed which is lasting for nearly three hours, costing time and data bandwidth to him/ her. Alternatively, neither he/she would be interested in manually browsing the video timeline to look for the particular goal event. Here, a football goal event seeker is a target user. More commonly it could be anyone, e.g. seeking fashion model ramp wall accident events, an Olympic inaugural torch lighting event, a movie action sequence, a road accident event, etc. Thereby there is need to present a solution which can present only the video event extraction related to interest of the user. The research problem definition as the author is building-up here is the precise event extraction from an unconstrained video feed.

Furthermore, the precise video event extraction could be made possible with help from semantic based content identification. For a video, it comprises of three levels of information. These are raw video data, low level features and semantic contents. The raw video data would constitute the attributes like frame rate, video length, format, etc. The secondary low level feature information would be of audio, text and visual features, such as colours, shape distribution and motion etc. The last and the highest level of information content would be the semantic contents of events recoded in the video. These semantic levels would be visually appreciated by the humans. For a researcher looking for precise event extraction means the semantic based even classification of the video frame sequence and then identifying and extraction of the particular event. The semantics based content search in the video is not that straightforward problem. Many researchers across the world had been working out multiple methods. The methods differ in the manner the video information is being worked out. It could be purely audio based or audio aided frame sequence extraction $^{\frac{1-3}{3}}$, or visual object motion based extraction. For example, an audio based video extraction would simply grep out the "Goal" sound in the video audio and then track out the corresponding frames in neighbourhood of matching timestamps from the video frame sequence. Unfortunately, many of such techniques won't yield fruitful or successful results. For example, it's not necessary that the audio information would be present always in the video. Besides as literature shows, many of noteworthy researchers ${ }^{4}$ had also worked on ontology based semantics information extraction. Where they primarily look for key frames extraction based on object identification and then workout different topological relations aligning to identification of an event from pre-determined object-event ontological classifications. Moreover, the problem becomes trickier since in many of the times, a video would be a frame sequence without having the direct relation to its semantic contents.

One can easily understand that the object extraction is one of most crucial components in the framework, since the objects are used as the input for the extraction process. Object detection from a static picture frame or a video sequence has attracted attention of many engineers working in the field. Photo cameras and the ones embedded in cell phone cameras can detect the face, smiles, mountains etc. already. The technology is well established. However, when it comes to not only detect the object but identify it as of certain product class, is the area where improvement is going on. The precise object identification comes through, improvising the object detector with multicue optimizer. Such a multicue optimizer would look for additional information from the video frames associated with the detected object. The objects shape and size are the very basic cues to look for. Moreover such additional information may include the detected object position in the frame. It can also be its association with the human appearance in the frame. Not all the time, in all frames, the object can be totally visible. The object detector has to work through occlusions at times. In such situations, it's not enough to look for additional cues from the same video frame. Thereby, an improvised system shall also look at the path traced by the object in the multiple frames in sequence and its association with the reference subject. The reference subject could be the human, vehicle or anything like that relating to the object. This is what a spatio-temporal cue does.

Present paper is targeted to propose and establish the multicue object identifications and inter-object spatio-temporal relation based semantics derivations for event identification and extraction from an unconstrained video sequence. As the authors are still 
working out in building the final automatic video event extraction system, the test initial results and system performance are encouragingly positive and precise. Thereby, this paper intends to present the proposal of an automatic precise video event extraction system concept with detailed discussion on literature and proposed system architecture with nascent indicatory results, surpassing the precision levels of other similarly reported out state of the art systems among recent literature.

The downstream sections summarize the key ideas from the related research works as reported in literature. The proposed system works out the video extraction based on precise object identification and then the spatio-temporal cues from multi object scenario. Thereby authors had tried to bring in the relevant efforts from literature for object identifications, and video extractions techniques for collected and classified glance at them in this section of the article.

The baseline idea begins with the thought process of considering the unconstrained video as the quasi-steady sequence of frames capturing the live event or happening. Thereby, each frame is likely to have multiple objects appearing in itself, whereas, most of these objects are bound to appear in the frame defining the target event. The individual object detection from the video frame sequence had itself been a separate research area. And so multiple of techniques for object detection from vide can be found reported in literature. The researchers working in the domain of video event extraction obviously started with object detection and its identification with specific class. Thereby, one can naturally appreciate the notion of even capturing, by detection of multiple objects in video frames and then their tracking of their appearances in subsequent frames. Going further, it's not only the objects appearances, but also tracking their relative positions in frames by frame manner. This explains the terminology "spatio-temporal" object tracking in video. Below Table 1 summarizes some of the key efforts for video based object detection and identification at times.

Table 1. Summary of generic object detection techniques from literature.

\begin{tabular}{ccl}
\hline Authors & Year & Description \\
\hline 6 & 2000 & $\begin{array}{l}\text { Proposed a method to learn heterogeneous } \\
\text { models of object classes for visual } \\
\end{array}$ \\
& recognition.
\end{tabular}

8

2006 Large-scale evaluation of an approach that represented images as distributions (signatures or histograms) of features extracted from a sparse set of key-point locations and learnt a Support Vector Machine classifier with kernels based on two effective measures for comparing distributions.

2007 Approach considered object categorization and figure-ground segmentation as two interleaved processes that closely collaborate towards a common goal. The resulting approach can detect categorical objects in novel images and automatically infer a probabilistic segmentation from the recognition result.

2008 Proposed a robust fast global motion estimation (GME) method for video object segmentation

2009 Object detection system based on mixtures of multi scale deformable part models. Their system relied heavily on new methods for discriminative training of classifiers that make use of latent information. And also on efficient methods for matching deformable models to images.

2010 Proposed moving object detection algorithm that used motion clustering and classification from only two consecutive image frames captured by a free-moving camera.

2011 Proposed a moving object detection approach for occluded objects in a video sequence with the assist of the SPCPE (Simultaneous Partition and Class Parameter Estimation) unsupervised video segmentation method.

2012 Converted a supervised segmentation problem into an unsupervised one using occlusions as the supervision mechanism. As a result, they had a fully unsupervised method for detecting and segmenting an unknown number of objects. 
It would be of readers help to mention about the work by researchers ${ }^{14}$, who presented a comprehensive survey of audio based video event extraction techniques from literature. They stressed on how the video event detection has been one of vital research area in computer vision. Although their major survey focused on detailing the works about video extraction based on audio cues. Moreover they concluded that there is still large scope of event extraction possible making use of multiple of video informatics.

\subsection{Video Object Detection Using Spatio- Temporal Cue and Similar Information}

While a large group of researchers focused on utilizing the primary video informatics, such as audio tracks, frame rates, frame colors etc., a new trend in the field was introduced where the event detection, or for that matter firstly the object detection from video was soon being achieved by spatio-temporal cues from the detected objects information. And thus the part of research or can be said as a fresh problem of optimum object detection was being worked out. Many of the early efforts indicate that the initial object detection systems assumed the object detection problem from a steady or static image. Particular object detection was cued by the multiple objects identified in the frame. And thereby tracking their mutual appearance cues constitutes the spatio-temporal system principle.

Objects as detected in video frames usually assigned to different object classes. However due to lack of holistic object class modeling many of times the objects identifications are not sufficient. In such scenarios researchers tried to address the problem by pairing of objects. Likewise, in 2004, researchers $\frac{15}{}$ in their paper proposed an approach for discriminative learning for implementing the pair wise constraints for object identification. One can see multiple benefits of this approach over the conventional trends. Firstly, in such a methodology, the object classification is driven by direct modeling of decision boundaries. To add more on benefits, their method also reduced the computational requirement by a considerable amount by combining the object pair wise constrains with the object labels, and thus making the process faster compared to the prior art by the time. The mainly demonstrated the system functionality over a surveillance video problem which was targeted for persons classification from the video.
One can easily appreciate the fact that success of an accurate spatio-temporal system would depend heavily on the exact object detection system. Therefore, more and more researchers group consistently came up with more and more accurate object detection systems. In 2005 , researchers ${ }^{16}$ showcased a unique innovative object detection system. Their system employed the approach of joint representation of pixels from the image frame. It's can be very well understood that such an approach permits the explicit modeling with local spatial image structure thereby. With a filtering kind of method, the frame background could be represented by single pattern segregating the embedded objects to detect the probabilistic. Additionally, their system employed the criterion for temporal perseverance. The image frame was segregated in multiple background and foreground layers for the objects in sight. This facilitates for their functionality of log likelihood in order to determine or classify a particular confusion pixel among the object or out of it. This inherently enhances the object tracking mechanism as whole while passing frame by frame operations. Moreover such a layered system actually introduced the coherent modeling ability to literature and can be considered as one of such pioneering efforts. As expected thus, such a system proved its efficacy in many of situations which are quite challenging for object detection due to obstructive interferences of multiple objects.

For real time videos one expects the presence of multiple objects with their highly complex appearance patterns in frame sequences. Many of times the backgrounds and object pixels are confusing for classifications. Similarly, there are other problems like in a spatio-temporal object tracking system, its doubtful that the system might actually tracing the path of the exact particular object consistently and not confusing among the multiple of objects. The objects appearances in multiple frame layers, or shall say, appearance with occlusions make the task demanding, both accuracy wise and also computationally. Systems reported among literature which followed a very conventional complex algorithm for resolving such issues had to come up with huge computational cost penalty. And still such specialized methodologies are witnessed to be non-robust. In 2008 researchers ${ }^{\frac{17}{7}}$, introduced an effective solution for this problem with a concept called "tracking scene". In this method, a scene is constituted of many of objects, including the target objects, which appear simultaneously in the frame sequence. Such objects, they claimed to be the ones identified through data mining 
techniques apart from the primary object detectors. And so the obvious properties or such secondary objects are that they are easy to track being mostly non-fractional or occlusion free appearance, and they do appear simultaneously with the target object, also have a similar motion patters, or shall say, common motion patter with that of the target object. Such a system would reduce the burden of heavy computational requirement due to sophisticated object detection systems.

The video based object detection can have multitude of practical and industrial implications of use. One of such application is for smart television. The televisions technology has seen a high rate of improvement in recent few years, from color television to flat screens, to internet enabled televisions of today. Very recently, researchers ${ }^{18}$ mentioned next generation smart television in their research article about multicue optimized object detection for smarter TV's. The idea basically states about the interactive television which allows user to get notified about the multiple objects appearing in a television program. This has its implication in TV based consumer market creation. The user would be made available or prompted with the commercial products/objects buying information. However, the essential step in realizing such a smarter television system is of course the most accurate object detection from an unconstrained video shoot being played on the television. Such a video footage can be from a movie or daily soaps or can be sports or for that matter a fashion walk. The accurate object detection was thus achieved by them $\frac{18}{18}$ with help of multi optimized object detection. It primarily employed three different cues for the object to be classified under particular required object class. These cues are appearance cue, topological cue and spatio-temporal cue. The appearance cue related to objects appearance in color and shape patterns. The topological cue relates to objects co appearance with other objects in frames and their mutual relative positions, shapes etc. The last, spatio-temporal cue relates with path traced by object in a dynamic frame sequence with reference to other co-appearing objects. In the current work, authors had leveraged the multicue optimized object detection system from this work $\frac{18}{}$.

\subsection{Video Event Extraction}

There are many of literature published in this area of video event extraction so far. An overlook at the relevant literature articles over a decade reveals that the newer and fresh ideas are continuously pouring in with more enhanced technologies offering high performance systems. As one scans through the relevant literature, the overall idea of the working principle, or the basis of event identification in a video frame sequence can be categorized in few major classes. These are:

- Event identification using video informatics;

- Event identification using object detection and their spatiotemporal cues; and

- Event identification based on ontological derivatives.

At the earliest efforts were merely of defeating the challenges of object detection type as been reviewed in previous section. Later researchers devised several other aspects of a video to make use as handle to detect and identify the relevant event. This video file information is in form of low level audio tracks and its further derived components such as frequencies etc. Also the information of graphical content, color specs etc. had been also used by some of researchers. However, the implications of such ideas could only be useful for certain predetermined events and so the scope was not generalized for a random user experience. In more recent times, the rise of ideas such as Video Semantic COntent Models (VISCOM) and other ontological based approaches auto defining the events are seeing the future of this problem.

As early as in 1998, researchers ${ }^{19}$ presented their system which was originally developed for more of surveillance purpose. They tried to address the objectives of human object identifications and also the actions represented by the human or group of humans from surveillance footage. However, this method can easily be identified with its limitations of being pre assumptions based to detect certain specific actions only. One has to appreciate their efforts as one of the very starting ideas in the field of video based event extraction.

Many of researchers also attempted to identify the event based on objects detection and the simultaneous detection of the objects dynamics motions frame by frame in the video. Researchers ${ }^{20}$ presented a system for event detection and analysis from video streams. Their approach was based on a detection and tracking module which used to extract moving objects trajectories from a video stream. These trajectories, together with a rough description of the scene, were then used by the behavior inference module in order to recognize and classify object motion. The hierarchical tasks are performed on a buffered set of frames in order to provide accurate 
results by taking into account the temporal coherence of moving objects.

Video event extraction basically also relates with the image processing branch of research in computer sciences. In prior work ${ }^{21}$ authors had demonstrated that use of CUDA can provide with a novel massively dataparallel general computing method, also being cheaper in hardware implementation at the same time. Going further, author ${ }^{22}$ recommended a system that would search for relevant video using color feature of video in response of user Query. Thereby proposed systems facilitated the segmentation of the elementary shots in the long video sequence proficiently. In the proposed process, the extraction of the color features using color histogram, color moment and color coherence vector is performed and the feature library is employed for storage purposes. Moreover, the system was also updated with the facility to user to not only search for a relevant video but also to register his/her videos on the web server.

The use of spatio-temporal cues for the objects in video for event frame identification has been often claimed as a definitive technique of video event extraction. And thus such attempts are very popularly observed to be reported in literature $\frac{23-25}{}$. To add to the list of ontological framework based systems, researchers $^{26}$ presented a top level ontology which provides a framework for describing the semantic features in video. In their system, at first, they identify the key components of semantic descriptions like objects and events and how domain specific ontology can be developed from them. Thereafter, they present a set of classes defining the event predicaments. Successively, then they develop schemes for reasoning the captured event to specified event class as needed. Thus they demonstrated the system implementation for predefined event classes based on developed ontological framework. However, the simultaneous implications of object detection based spatio-temporal cues and ontological frameworks are rarely seen. Few efforts tried to make combined use of spatio-temporal cue and highlevel semantic content in terms of object. They have mainly focused on demonstrating the system structure based on VISCOM. Their work forms the basis of current paper too.

Moreover, some of the researchers ${ }^{27}$ had also reported the use of Latent Semantic Indexing (LSI) in association with Markov Semantic Indexing (MSI) to successfully demonstrate the improved results over the state of the art. In another noteworthy work ${ }^{28}$ summarizes the survey of methods for practical and potential applications of ontology in the field of software engineering. As the video-event complexity grows with growing system demand and the datasets because of more and more generalizations of requirement, a class of researchers also opting for employing fuzzy systems for defining the complex semantic based ontological framework, which is to be used for accurate and more general event extraction systems. To make a note of such efforts one can refer to the noteworthy works $s^{29,30}$.

\section{Proposed System}

The system architecture of video event extraction system based on multicue object identifier and spatiotemporal inter objects relation based event semantics is illustrated in Figure 1(a). The underline semantics modeling based event extraction has been inspired from prior literature ${ }^{4}$. The Figure 1(b) shows the existing system as reported by earlier researchers It can be understood that in current efforts authors are making a proposal of enhancing the primary object detection system for the improved accuracy of the final overall system. Thereby, the key contribution of this paper is the proof of concept of usage of multi-cue optimized object detector with spatio-temporal cue and VISCOM model as demonstrated in detailed in literature ${ }^{4}$. The present section will focus on introducing the multicue object detection framework into the video event extraction system as augmentation, and thus would see the details of multicue object detection system in its own. The details of VISCOM modeling and complexity therein can be found in the referred and the same has been avoided for repetition and scope purpose.

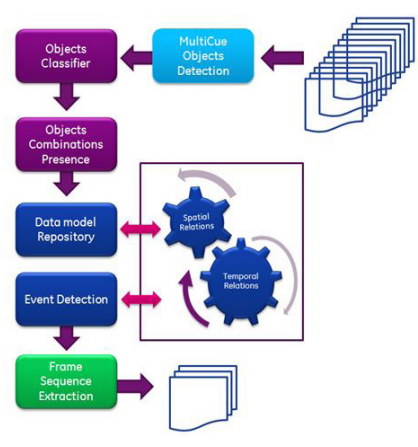

(a)

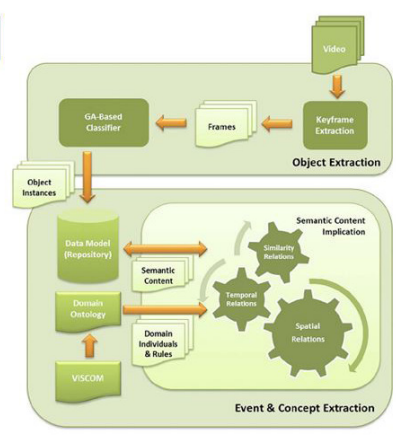

(b)
Figure 1. Proposed and existing system architecture. 
The multicue object detection system has two-fold reasons of applicability here. First, the multicue object detection would amplify the system capability to exact object detection as it is entitled for. Secondly, the spatiotemporal cue as employed in multicue object detection for the singular or individual object for identification of itself, would also have the additional benefit of resolving the inter-object spatio-temporal status quo and thereby impart a lot of simplicity to the overall system algorithm as such.

Ideologically the multicue object detection system is also leveraged from another original work ${ }^{18}$.

Figure 2 shows the architectural block diagram of the multicue object detection system as had been plugged into the video event extraction system. As can be seen from Figure 2, the system chunks the live input video footage into sequence of frames. A coarse or relaxed object detector is employed to tag the frame wise objects appearance. The primary object detector is relaxed in terms, so that it can identify as many possible objects as possible from the frame. These will be later subjected to multi-cue optimizer to detect out the exact objects pertaining to desired target class. Moreover, using relaxed object detector helps in lowering the misses, even though there might be some possible false identification. The function of primary object detector is to tag the objects from frames which relate to desired and predefined product class. So that it provides their feature vector representations.

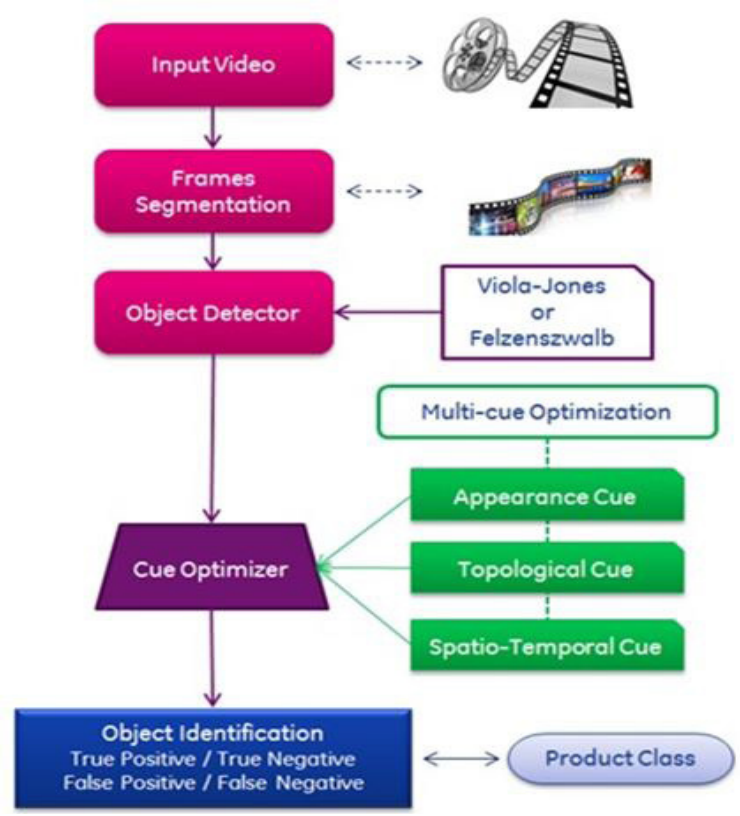

Figure 2. Illustration of multicue object identifier.
Thereafter, a multi-cue optimizer works on the tagged objects and their feature vectors as provided by primary object detector. As originally introduced $\frac{18}{}$, the multicue optimizer detection technique is a generic one and not related to specific object class detection. One can use the same to detect different objects. Moreover, the multicue optimizer technique works perfectly for any random different videos. It means it need not be a fashion video always; it could be a movie or tele advertorial, etc. Thereby one has to appreciate that the technique does not make any pre assumptions about sequential positions of objects in the videos. So the object can appear in any random part or corner of a frame dynamically would be treated irrespective of it. Most important part of everything is this multicue optimizes, as the name suggests, employs three cues to aid up the information for associating the detected object to a target class. These cues are - appearance cue, topological cue and spatio-temporal cue. The overall algorithm of the system is similar to as laid out recent literature advances ${ }^{\frac{18}{}}$. And so won't be of reader's interest to repeat the same here again. However, overview and formulation of different cues as used within multicue optimizer are presented as follows.

- Appearance cue - Basically, the appearance cue is the direct measure of the detected object $\left(O_{i}\right)$ of being the target class $(C)$. So mathematically it can be represented as a probability $P(O / C)$, where $\mathrm{I}$ is the $\mathrm{i}^{\text {th }}$ appearance. This probability is obtained from a trained object detector. Any object detector can be trained to identify the detected object belonging to the specific target class and used for the purpose. Additionally, the trained object detector has to perform two more duties. First, it has to also return the object detection box size as well as its position in the frame. Secondly, it shall also confirm that the identified detected object is correlating with its appearance in the previous frame appearance. This is to ensure the object identity. In the system this is defined as $\Omega$ as follows:

$$
\Omega\left(O_{i}, O_{j}\right)=s\left(\tau\left(O_{i-1}\right), \tau\left(O_{i}\right)\right)
$$

Here, $\tau$ is the feature vector representation of the object and $s$ being the function representing the similitude between these feature vectors at $i^{\text {th }}$ and its previous appearance.

- Topological cue - Topological cue is to ensure the detected objects relative position in the video frame. For example, suppose the target class is a necklace. 
Then the detected object, i.e. the box shall be in close vicinity of the models face. Similarly, if the object class is bangle, then the detected object box shall be in certain perimeter range from the model face. Mathematically this can be presented as:

$$
\Psi\left(O_{i}\right)=\frac{d_{l}\left(l\left(O_{i}\right), l\left(F_{i}\right)\right)}{b_{m}}
$$

Here, $l$ represents the position of the detected object $\left(O_{i}\right)$ and model's face $\left(F_{i}\right)$ in the very same $\mathrm{i}^{\text {th }}$ frame. Moreover, $d_{l}$ is the measure of geometrical distance between the two, i.e. detected object and the model's face. Further, $b_{m}$ being the set expected distance as per the target class, $\psi$ represents the comparatively assessed relation between $d_{l}$ and $b_{m}$. Thus the topological cue takes care of expected appearance position information of detected object call to ascertain its identity.

- Spatio-temporal cue - This can be understood as an advancement of the concept of topological cue. As explained the topological cue checks out the relative distance between the detected object and the related object, say, models face. Spatio- temporal cue sets up the variation limit of his distance for sequential appearance of the object, frame by frame. Thus the spatio-temporal cue evaluated as:

$$
\Gamma\left(O_{i}, O_{j}\right)=\frac{\min \left(d_{l}\left(O_{i}, O_{j}\right), d_{l}\left(R_{i}, R_{j}\right)\right)}{\max \left(d_{l}\left(O_{i}, O_{j}\right), d_{l}\left(R_{i}, R_{j}\right)\right)+\epsilon}
$$

Here, $\varepsilon$ is a small infinitesimal constant introduced to avoid the zero divisor error. Thereby the spatio-temporal cue measure, $\Gamma$, would trace the detected objects within frame position path temporally.

Towards the end, the system does segregate the detected object results as:

- True Positive: Object (Shoe) detected,

- True Negative: No Object (Shoe) detected,

- False Positive: Object (Shoe) is present but not detected, and

- False Negative: Object (Shoe) is not there but detected.

\section{Test Results}

The spatio-temporal cue is one of the prime key of the current multicue system. It is well understood from previous section that spatio-temporal cue actually relates the topological information, i.e. objects relative position in dynamic or frame to frame manner as time evolution. The semantic models can be built up by defining the certain spatio-temporal relations demonstration by the multiple objects making their appearance simultaneously in the given frame.

The video event extraction system thus built has been tested with two scenarios. The testing results are presented here in this section. The first case is where target is to extract the exact event footage from the football match video. There are many of football match recorded videos as available from internet are being used for the case purpose. Whereas, in second case the target is to extract the event related to the fashion model ramp walk accidental fall from a fashion show video. Figure 3 and 4 demonstrate the event extraction in the two mentioned test cases with the help from actual frames as captured during the program run.

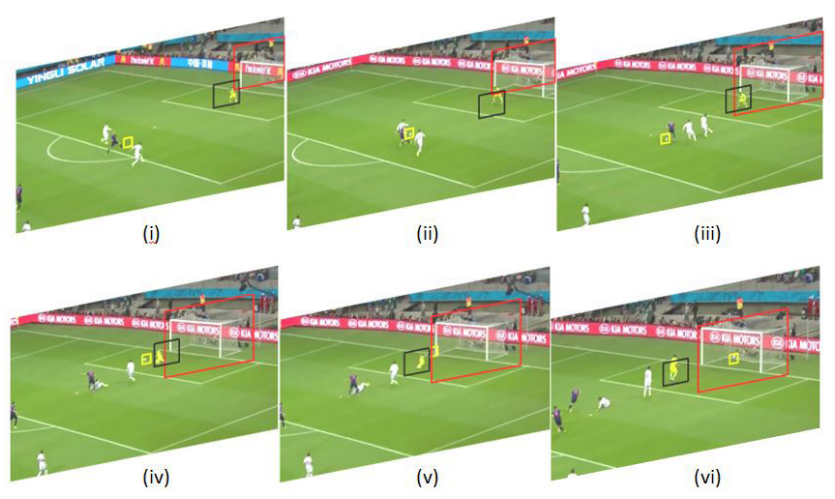

Figure 3. Event extraction demonstration: case-1.

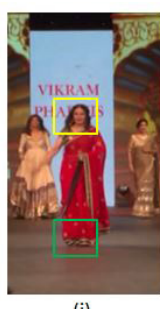

(i)

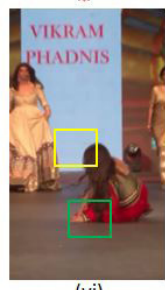

(vi)

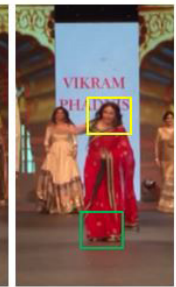

(ii)

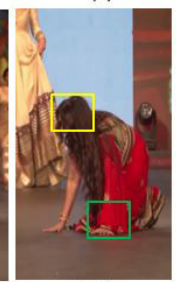

(vii)

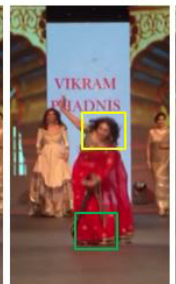

(iii)

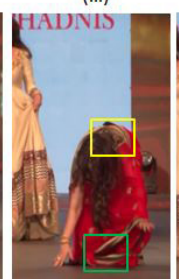

(viii)

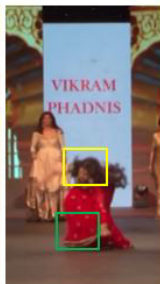

(iv)

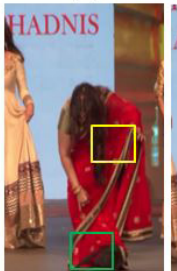

(ix)

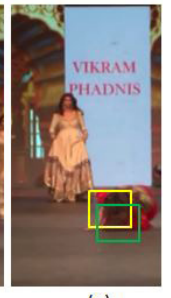

(v)

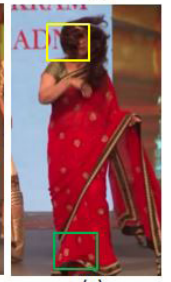

(x)
Figure 4. Event extraction demonstration: case-2. 
In first case, the event of 'goal' has the semantics definition based on the spatio-temporal conditions where, the video frames are scanned in sequence to detect the simultaneous appearance of the objects from pre-defined object class, 'ball', 'hoop' and the 'goalkeeper'. Wherein, the temporal paths of hoop and the ball are being tracked by the multicue object identifier automatically, the data model engine keeps a check on their relative distancing. So that, as the three objects, 'goalkeeper', 'ball' and the 'hoop' appear at the same time where the spatio-temporal paths of object 'ball' and the object 'hoop' meet or cross for a threshold frames number, indicating that their relative distance going to zero and the two object boxes overlapping on each other. Figure 3 demonstrates this with the help from actual extracted frames with object identifiers. This is when the event 'goal' is identified. The system thereby then extracts out the fixed number of fames upstream of the identified event frame and downstream of it to compile the extraction footage from the video.

In the second case, the similar semantic model for the event describing the fall of fashion model during ramp walk, is defined by the spatio-temporal relation between the objects detected, 'model face' and 'model shoes'. Thereby the multicue object identifier hunts for object classes of model faces and shoes. When the accident happens, the human model leans down in action slowly, frame by frame, so that the face comes in close circle to feet or the shoes. Figure 4 demonstrates this with the help from actual extracted frames with object identifiers. Thus the accidental fall event gets identified and extracted, in very similar manner as elaborated in the case- 1 description.

The precision and recall are the two measures to quantify the success rate of system. These are the commonly reported parameters for such systems as observed from literature. Precision means the fraction of true positive objects detections to overall object detection. Whereas recall means, fraction of true positive object detection to actual expected true positive object detection. For the present work this can be formulated as:

$$
\begin{aligned}
& \text { Precision }=\frac{\text { True positive detections }}{\text { Overall detections }} \\
& \text { Recall }=\frac{\text { True positive detections }}{\text { Actual expected True positive detections }}
\end{aligned}
$$

It might be early to calculate the precision and recall measures for the testing results alone. However, as the testing was tried out over the two events of 'goal' and 'model fall' with 3 sample videos each, in the absence of any post-processing automation, the precision-recall statistic has been manually calculated by pre-identifying the individual event counts in each test videos. The performance outcome has been tabulated in Table 2 .

Table 2. System performance.

\begin{tabular}{lcccc}
\hline & \multicolumn{2}{c}{ Event - Goal } & \multicolumn{2}{c}{ Event - Model Fall } \\
\cline { 2 - 5 } & Precision & Recall & Precision & Recall \\
\hline Video 1 & 0.9045 & 0.8422 & 0.9167 & 0.8369 \\
Video 2 & 0.9467 & 0.9063 & 0.8915 & 0.8014 \\
Video 3 & 0.8842 & 0.8113 & 0.9637 & 0.8947 \\
\hline Average & $\mathbf{9 1 . 1 8} \%$ & $\mathbf{8 5 . 3 3 \%}$ & $\mathbf{9 2 . 4 0 \%}$ & $\mathbf{8 4 . 4 3 \%}$ \\
\hline
\end{tabular}

It inferences that, the current implemented multicue optimized object detection system has average precision of $91.79 \%$ and average recall of $84.88 \%$, which is definitely better than findings reported in literature so far. ${ }^{4}$

\section{Conclusion}

The paper presents a proof of concept of employing a multicue optimization based object identifier for a system of automated precise event extraction from an unconstrained video frame sequence. The reconstructed video extraction system employs VISCOM as reported in the literature. The author's idea is to further enhance the system accuracy and performance by using a multicue optimization based object detector instead of reported simple primary object detector. The benefits of multicue optimization object identifier bring in the additional aid of spatio-temporal cue based calculation and the similar cues for multiple identified objects in the video can be used to identify their co-occurrence and a resultant event, which can be as defined by the VISCOM model engine as well. The overall system is obviously expected to have an edge over the existing and reported system in literature in terms of performance accuracy.

The authors had reconstructed the baseline building blocks of literature reported VISCOM based system and integrated the multicue optimized object identifier into it. For the testing of the concept expectations, the system was tested with six videos, to extract two events, namely, a goal in football match and fall action of fashion model on the ramp. The primary performance measures of precision and recall had been presented along with the 
spatio-temporal cue demonstration for both video types. The overall results of the system thus proposed and demonstrated is found to be better than state of the art systems from literature.

\section{References}

1. Gelin P, Wellekens CJ. Keyword Spotting Enhancement for Video Soundtrack Indexing, ICSLP, IEEE Fourth International Conference on in Spoken Language. 1996; 2:586-89.

2. Saraceno C, Leonardi R. Audio as a Support to Scene Change Detection and Characterization of Video Sequences, IEEE International Conference on in Acoustics, Speech, and Signal Processing. 1997; 4:2597-600.

3. Tseng VS, Su J, Huang JH, Chen CJ. Integrated Mining of Visual Features, Speech Features, and Frequent Patterns for Semantic Video Annotation, IEEE Transactions on Multimedia. 2008; 10(2):260-67.

4. Yildirim Y, Yazici A, Yilmaz T. Automatic Semantic Content Extraction in Video using a Fuzzy Ontology and Rule based Model, IEEE Transactions on Knowledge of Data Engineering. 2013; 25(1).

5. Weber $\mathrm{M}$, Welling $\mathrm{M}$, Perona P. Towards Automatic Discovery of Object Categories, Proceedings of IEEE Conference Comput. Vis. Pattern Recognition; 2000. 2, p. 101-08.

6. Viola P, Jones M. Rapid Object Detection using a Boosted Cascade of Simple Features, Proceedings of IEEE Comput. Soc. Conf. Comput. Vis. Pattern Recognition (CVPR); 2001. 1, p. 511-18.

7. Zhang J, Marszalek M, Lazebnik S, Schmid C. Local Features and Kernels for Classification of Texture and Object Categories: A Comprehensive Study. Proceedings of Conf. Comput. Vis. Pattern Recognition Workshop (CVPRW); 2006. p. 13.

8. Leibe B, Leonardis A, Schiele B. Robust Object Detection with Interleaved Categorization And Segmentation, International Journal of Computer Vision. 2007; 77(13):259-89.

9. Qi B, Ghazal M, Amer A. Robust Global Motion Estimation Oriented to Video Object Segmentation, IEEE Trans. Image Process. 2008; 17(6):958-67.

10. Felzenszwalb PF, Girshick RB, McAllester D, Ramanan D. Object Detection with Discriminatively Trained Part-Based Models, IEEE Trans. Pattern Anal. Mach. Intell. 2010; 32(9):1627-45.

11. Kim J, Ye G, Kim D. Moving Object Detection Under Free-Moving Camera. Proceedings of 17th IEEE Int. Conf. Image Process (ICIP); 2010. p. 4669-72.
12. Liu D, Shyu ML, Zhu Q, Chen SC. Moving Object Detection Under Object Occlusion Situations in Video Sequences. Proceedings of IEEE Int. Symp. Multimedia (ISM); 2011. p. 271-78.

13. Ayvaci A, Soatto S. Detachable Object Detection: Segmentation and Depth Ordering from Short-Baseline Video, IEEE Trans. Pattern Anal. Mach. Intell. 2012; 34(10):1942- 51.

14. Natarajan R, Chandrakala S. Audio-Based Event Detection in Videos - A Comprehensive Survey, International Journal of Engineering and Technology. 2014; 6(4):1-12.

15. Yan R, Zhang J, Yang J, Hauptmann AG. A Discriminative Learning Framework with Pair-Wise Constraints for Video Object Classification, IEEE Trans. Pattern Anal. Mach. Intell. 2006; 28(4):578-93.

16. Sheikh Y, Shah M. Bayesian Modeling of Dynamic Scenes for Object Detection, IEEE Trans. Pattern Anal. Mach. Intell. 2005l; 27(11):1778-92.

17. Yang M, Wu Y, Hua G. Context-Aware Visual Tracking, IEEE Trans. Pattern Anal. Mach. Intell. 2009; 31(7):1195209.

18. Fleitis F, Wang H, Chen S. Enhancing Product Detection with Multicue Optimization for TV Shopping Applications, IEEE Transaction on Emerging Topics in Computing. 2015; 3(2):161-71.

19. Davis LS, Fejes S, Harwood D, Yacoob Y, Haratoglu I, Black MJ. Visual Surveillance of Human Activity. Proceedings of Third Asian Conf. Computer Vision (ACCV); 1998. 2, p. 267-74.

20. Medioni GG, Cohen I, Bre'mond F, Hongeng S, Nevatia R. Event Detection and Analysis from Video Streams, IEEE Trans. Pattern Analysis Machine Intelligence. 2001; 23(8):873-89.

21. Daga BS, Bhute AN, Ghatol AA. Implementation of Parallel Image Processing using NVIDIA GPU Framework, Springer-Verlag Berlin Heidelberg, Communications in Computer and Information Science. 2011; 125:457-64.

22. Daga BS, Content based Video Retrieval using Color Feature: An Integration Approach, Springer-Verlag Berlin Heidelberg, Communications in Computer and Information Science. 2013; 361:609-19.

23. Do“nderler ME, Saykol E, Arslan U, Ulusoy O, Gu“du“kbay G. Video: Design and Implementation of a Video Database Management System, Multimedia Tools Applications. 2005; 27(1):79-104.

24. Fan J, Aref W, Elmagarmid A, Hacid M, Marzouk M, Zhu X. Multiview: Multilevel Video Content Representation and Retrieval, J. Electronic Imaging. 2001; 10(4):895-908.

25. Fan J, Elmagarmid AK, Zhu X, Aref WG, Wu L. Classview: Hierarchical Video Shot Classification, Indexing, and Accessing, IEEE Trans. Multimedia. 2004; 6(1):70-86. 
26. Nevatia R, Natarajan P. EDF: A Framework for Semantic Annotation of Video. Proceedings of 10th IEEE Int'l Conf. Computer Vision Workshops (ICCVW '05); 2005. p. 1876.

27. Sangeetha M, Anandakumar K. Annotation based Image Retrieval System by Mining of Semantically Related User Queries with Improved Markovian Model, Indian Journal of Science and Technology. 2015; 8(35):1-8.

28. Bhatia MPS, Kumar A, Beniwal R. Ontologies for Software Engineering: Past, Present and Future. Indian Journal of Science and Technology. 2016; 9(9):1-16.
29. Nikam P, Nandwalkar BR. Video Semantic Content Extraction using K-Means and Fuzzy Ontology, International Journal of Modern Trends in Engineering and Research. 2015; 2(7):129-33.

30. Shoba G, Kalaitchelvi S. Extraction of Video Semantic Content using Fuzzy Rule based Model, International Journal of Engineering Research and Technology. 2013; 2(12). 